\title{
Enquête sur la genèse du Deuxième quatuor à cordes de Michael Finnissy
}

\section{Amanda Bayley}

Traducteur : Jean-Michel Magniez

\section{OpenEdition}

Journals

Édition électronique

URL : http://journals.openedition.org/genesis/329

DOI : 10.4000/genesis.329

ISSN : 2268-1590

\section{Éditeur :}

Presses universitaires de Paris Sorbonne (PUPS), Société internationale de génétique artistique littéraire et scientifique (SIGALES)

\section{Édition imprimée}

Date de publication : 10 octobre 2010

Pagination : 37-54

ISBN : 978-2-84050-711-6

ISSN : 1167-5101

Référence électronique

Amanda Bayley, "Enquête sur la genèse du Deuxième quatuor à cordes de Michael Finnissy », Genesis [En ligne], 31 | 2010, mis en ligne le 21 septembre 2012, consulté le 19 avril 2019. URL : http:// journals.openedition.org/genesis/329; DOI : 10.4000/genesis.329 


\title{
Enquête sur la genèse du Deuxième quatuor à cordes de Michael Finnissy
}

\author{
Amanda Bayley
}

$\mathrm{C}$

ETTE ÉTUdE PROCÈdE D'UNE COLLABORATION avec le Kreutzer Quartet, subventionnée par un fonds de recherche de la British Academy. Notre projet, l'identification de nouvelles procédures et méthodes de recherche sur l'interprétation musicale en quatuor, impliquait un travail approfondi sur plusieurs œuvres du répertoire moderne et contemporain : Stravinsky, Ligeti, Lutosławski... ainsi qu'une nouvelle partition, dont l'appropriation par le quatuor, mais aussi la démarche compositionnelle, seraient documentées sur le vif1.

Pour rendre compte du processus créateur à l'origine du Deuxième quatuor à cordes de Michael Finnissy (2006-2007), nous avons rassemblé un certain nombre de « notes de terrain », rédigées à partir d'observations et d'entretiens, permettant d'échafauder une intrigue - une narration thématique - relative à l'évolution du morceau. Nos entretiens avec Finnissy, étalés sur un peu plus de deux ans et traitant de différentes étapes de la composition, ont fourni le matériau principal de la présente étude (voir Tableau $n^{\circ} 1$ ). Aux transcriptions de ces entretiens s'ajoutent celles des conversations entre le compositeur et ses interprètes, à l'occasion des répétitions. Outre le codage et la sélection de fragments parmi la profusion de données verbales associées à l'activité du compositeur, la démarche d'inspiration ethnographique ici adoptée s'accompagne de l'évaluation et de la mise en contexte des signes musicaux - qu'il s'agisse de parties instrumentales ou d'esquisses. Nous nous efforçons d'assurer un juste équilibre entre remarques analytiques et exigence d'adhésion aux points de vue du groupe étudié 2 .

Toutes les données figurant dans cette liste existent en format audio, mis à part les réponses (écrites) des instrumentistes au questionnaire et les échanges entre compositeur et interprètes (filmés). Après avoir initialement porté sur des questions générales à propos des sources d'inspiration de Finnissy et de sa conception de la pièce, les entretiens ont évolué vers des questions plus spécifiques, relatives, par exemple, à la manière d'exploiter son matériau ou à ses prises de décision concernant la notation. Chaque étape a immédiatement mis au jour des couches d'informations d'une importance cruciale sur le projet compositionnel, dont les notes et indications figurant sur la partition ne laissaient souvent rien soupçonner.

Il existe de nombreux commentaires publiés par les compositeurs sur leurs propres œuvres. L'avantage d'une approche basée sur des observations et des entretiens est qu'elle recueille de tels propos à différentes étapes du processus créateur et prend en compte les interactions entre le compositeur et les autres acteurs du processus en particulier ses interprètes. Les moments de répétition fournissent à l'analyste une matière non négligeable : ils révèlent les réactions du compositeur face aux questions spécifiques touchant l'interprétation. Ils expliquent aussi pourquoi il a décidé de noter telle ou telle chose et pourquoi il l'a fait de telle ou telle manière. C'est particulièrement vrai (même si ce n'est pas exclusif) pour tout ce qui touche au type de son qu'il souhaitait entendre ${ }^{3}$. Peu après la création mondiale de la pièce, une étape « réflexive » de

1. L'auteure tient à exprimer sa gratitude à l'université de Wolverhampton et au Conservatoire de Birmingham (Birmingham City University), qui ont financé la commande de l'œuvre.

2. Robert M. Emerson, Rachel I. Fretz et Linda L. Shaw, Writing Ethnographic Fieldnotes, Chicago, London, University of Chicago Press, 1995, p. 171.

3. Pour plus de détails concernant les répétitions ou d'autres aspects de cette recherche, veuillez vous reporter au site suivant, où sont indiquées les publications et exposés les résultats de recherches (y compris sous la forme de présentations multimédia des différentes exécutions et enregistrements de la pièce) : <www.wlv.ac.uk/sspal/stringquartetresearch $>$. 
Tableau $n^{\circ} 1$. Sources d'informations sur le processus compositionnel du Deuxième quatuor de Finnissy

\begin{tabular}{|l|l|}
\hline Éléments d'information & Dates \\
\hline $\begin{array}{l}\text { Entretiens avec le compositeur } \\
\text { Avant l'écriture } \\
\text { Avant les répétitions }\end{array}$ & $\begin{array}{l}10 \text { juillet } 2006 \\
4 \text { février } 2007\end{array}$ \\
\hline $\begin{array}{l}\text { Répétitions } \\
\text { Le Kreutzer Quartet avec le compositeur }\end{array}$ & 4 février 2007 \\
\hline Réflexion & \\
Réponse des interprètes à un questionnaire & Mars 2007 \\
\hline Discussions/interactions entre le compositeur et les interprètes & 23 mars 2007 \\
\hline Entretiens avec le compositeur & \\
Après l'exécution & 27 juillet 2007 \\
Sur esquisses & 8 mai 2008 \\
Sur esquisses & 26 juin 2008 \\
Sur esquisses & 26 septembre 2008 \\
\hline
\end{tabular}

notre étude comportait une exécution publique et une présentation de la pièce à la Royal Academy of Music de Londres, qui ont débouché tout naturellement sur de nouvelles explications de la part du compositeur en réponse à des questions du public et des interprètes. Au cours des étapes ultérieures de cette étude - la partition étant achevée -, les questions se sont centrées sur les esquisses : tandis que notre premier entretien avec Finnissy avait eu lieu avant qu'il ne s'engage dans la composition du quatuor, les suivants ont été menés dans la foulée de la création et se sont référés aux traces de son activité dans une période où sa mémoire en était encore fraîche.

En combinant ces différentes sources, on obtient un certain nombre d'indices et d'explications qui contribuent à une compréhension du projet compositionnel bien meilleure que celle que l'on peut tirer d'une étude de la notation. En réexaminant certaines questions particulières parmi celles soulevées au cours des deux ans qu'a duré cette recherche (2006-2008), on découvre comment des décisions relatives à certains aspects du morceau peuvent être mûries, voire réévaluées, au cours du temps, à différentes étapes de la conception, de l'écriture, de la réflexion et du travail sur l'interprétation.

Face à certaines questions du premier entretien, Finnissy nous a déclaré que l'heure n'était pas encore aux commentaires. Il en a pourtant fait quelques-uns. À la question concernant d'éventuelles influences directes sur sa pièce, il répond :
Il est vraiment trop tôt pour dire cela maintenant, même si mon expérience semble indiquer que c'est effectivement comme cela que je raisonne, la plupart du temps. Je ne me place pas en dehors de la tradition. Je n'essaie pas non plus - pas consciemment - de bâtir des ponts dans sa direction. Je me contente de reconnaître que j'en fais partie, parce que chacun de nous se rattache à une historicité, ponctuée d'événements mais aussi de changements ${ }^{4}$.

Le présent article est loin de pouvoir contenir toutes les remarques de Finnissy sur la tradition du quatuor à cordes et sur son propre processus compositionnel. À défaut de viser l'exhaustivité, ce compte rendu se fixe un quadruple objectif : tout d'abord, identifier quelques-uns des changements apportés par Finnissy à la conception originale de sa pièce ; deuxièmement, repérer quelques-unes des influences qui se sont exercées sur cette œuvre ; ensuite, expliciter la façon dont le compositeur exploite différents matériaux de base ; enfin, révéler de quelle manière, dans le cadre d'une étude de « terrain » comme celle-ci, les esquisses des compositeurs peuvent fournir le point de départ - et aussi répondre - à des questions sur le processus compositionnel5.

4. Entretien avec l'auteure, le 10 juillet 2006.

5. On pourra trouver d'autres informations concernant, en particulier, le deuxième et le troisième point ( $\mathrm{y}$ compris des transcriptions de fragments parlés) dans un article présentant l'application développée à partir de ce projet : Amanda Bayley et Michael Clarke, « Analysing Michael Finnissy’s Second String Quartet: A Multimedia Interactive Approach », musik. theorien der gegenwart (à paraître). 


\section{Matériaux de départ}

À notre question « Votre matériau de départ naît-il spontanément ou bien a-t-il une origine définie ? », Finnissy répond d'abord en répétant : «Il est beaucoup trop tôt pour le dire. » Toutefois, il poursuit en donnant une description assez expressive des images qui l'habitaient...

Ça peut paraître bizarre, mais il m'arrive effectivement d'avoir des visions, comme dans un rêve (même si ce sont probablement des rêveries plutôt que de véritables rêves), au cours desquelles je vois quelques mesures d'un morceau. Cette fois-ci, pour une raison que j'ignore, ces mesures ressemblaient beaucoup au premier quatuor de Schönberg. Je ne sais pas trop pourquoi. Sauf qu'elles ne sont pas dans la même tonalité. Apparemment, il s'agit d'une tonalité à bémols. Je crois avoir vu quelque chose comme cinq bémols à l'armure. Travailler à partir d'une telle idée a une incidence singulière dans le cas d'un quatuor à cordes, car les tonalités à bémols donnent un son particulier à la musique pour cordes. Voilà donc un concept assez intéressant. Maintenant, je ne sais pas du tout jusqu'à quel point je vais m'y tenir ni quel rôle ce concept va jouer $6[\ldots]$.

Je ne crois pas que j'utiliserai le premier quatuor de Schönberg car il est soumis à des droits d'auteur et je n'ai pas envie d'être obligé de payer. À ce stade, je ne peux pas dire. D'autant qu'assez vite, j'avais parlé à Neil [le violoncelliste] d'une autre idée. Je voulais... - je ne sais pas trop pourquoi j'avais décidé ça - ...j'avais envie d'ajouter des chants d'oiseaux au quatuor. En effet, ce qui m'intéresse, c'est notre façon d'écouter la musique ou notre façon d'écouter les sons. Ou plutôt, la manière dont, assez souvent, nous n'écoutons pas les sons. Et cette idée que les représentations que nous nous faisons des sons de la nature, d'une part, et de la musique, d'autre part, seraient, en quelque sorte «parallèles », sans se recouper forcément. Et je n'ai pas non plus l'intention d'intervenir sur ces chants d'oiseaux, de quelque manière que ce soit. Il n'est pas question de les transcrire, dans le style de Messiaen, ni de les rendre mécaniques à la façon de Granados. Ils seraient là, c'est tout ; un peu comme le bruit des chantiers et de la circulation, qui nous parviennent en ce moment, ici, dans Baker Street, pendant cet entretien. Ce serait un moyen d'attirer l'oreille du public vers le quatuor, par le biais d'autre chose. Mais, quant à savoir si je vais rompre la continuité des chants d'oiseaux, il est encore trop tôt pour le dire car cela s'inscrit aussi dans le processus de composition. Bien sûr, cela change pas mal de choses si l'on se met à interrompre un phénomène continu, donc, en l'occurrence, si le quatuor brise la continuité du chant des oiseaux, ce qui était prévu au départ. À l'heure qu'il est, je n'ai plus de certitudes?

Il apparaît que les intentions originelles de Finnissy se sont radicalement transformées, pour des raisons échappant à son contrôle et qu'il nous a confiées au cours de l'entretien qu'il nous a accordé sept mois plus tard :

Il me fallait pas mal de temps pour que ça se fasse. Novembre est arrivé et je n'avais toujours pas d'enregistrement d'oiseaux. Personne ne chantait dans le jardin. Il ne restait tout simplement pas assez de temps pour écrire une demi-heure de musique. J'ai donc dû reconcevoir entièrement le projet. Mais, pendant tout ce temps, je n'étais pas resté inactif. Je m'étais intéressé à un autre quatuor à cordes, dont on prétend qu'il a été conçu (vaguement) autour des chants d'oiseaux. Il s'agit de L'Alouette (on l'appelle aussi quelquefois le quatuor «Hornpipe », donc ce peut être aussi bien une alouette qu'un hornpipe). Quoi qu'il en soit, cela m'a amusé et j'ai persévéré dans cette idée. Mon intention première était de prendre le quatuor de Haydn à rebours, parce que je n'aimais pas la manière dont il démarrait. Pour moi, en tout cas. C'est parfait pour Haydn, mais pas pour moi. Je voulais une ouverture dans le genre presto. Le dernier mouvement de la pièce de Haydn se rapproche d'un presto. Alors, j'ai décidé de prendre le quatuor de Haydn à reculons 8 .

Ainsi, le finale vivace de Haydn (voir fig. 1a) a servi de base de travail aux vivace de Finnissy. Les similitudes de rythme et de texture sont patentes.

6. Finnissy a bel et bien gardé l'idée d'une armure à bémols (quatre bémols au lieu de cinq) mais uniquement dans une des parties de la pièce, à cause des changements survenus lors de la conception. La question des tonalités et des armures est revenue dans des entretiens ultérieurs. Nous en rendons compte à la fin de l'article.

7. Entretien avec l'auteure, le 10 juillet 2006.

8. Entretien avec l'auteure, le 4 février 2007. 


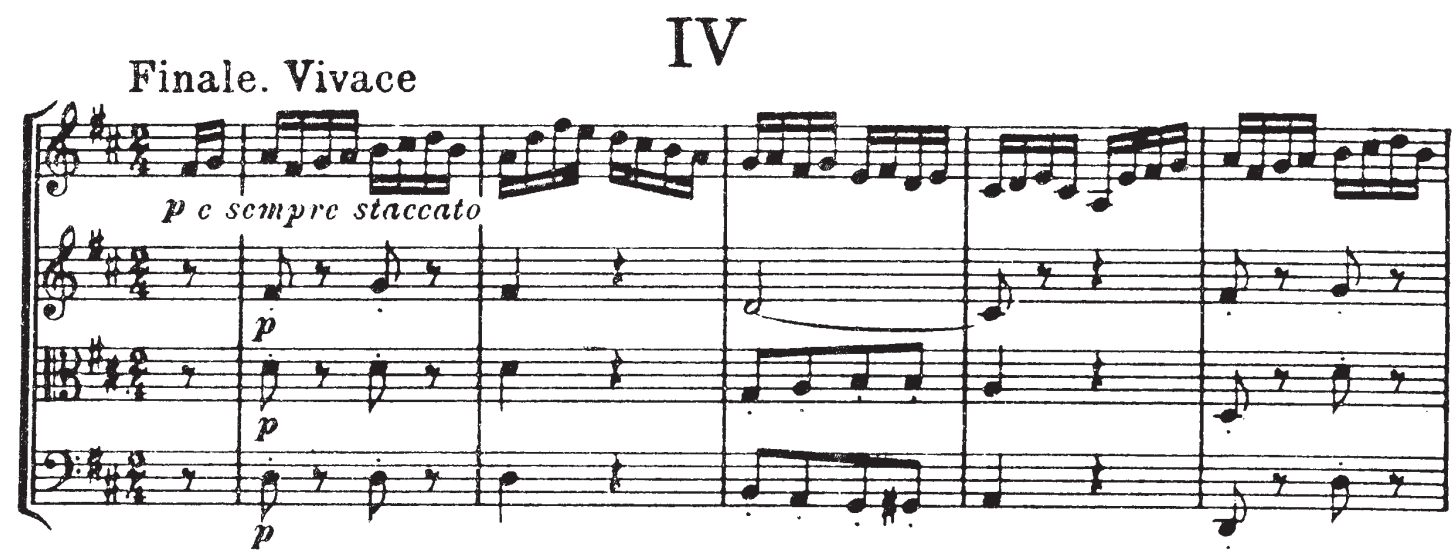

Fig. 1a : Ouverture du quatrième mouvement du quatuor à cordes de Haydn, opus $64, \mathrm{n}^{\circ} 5$

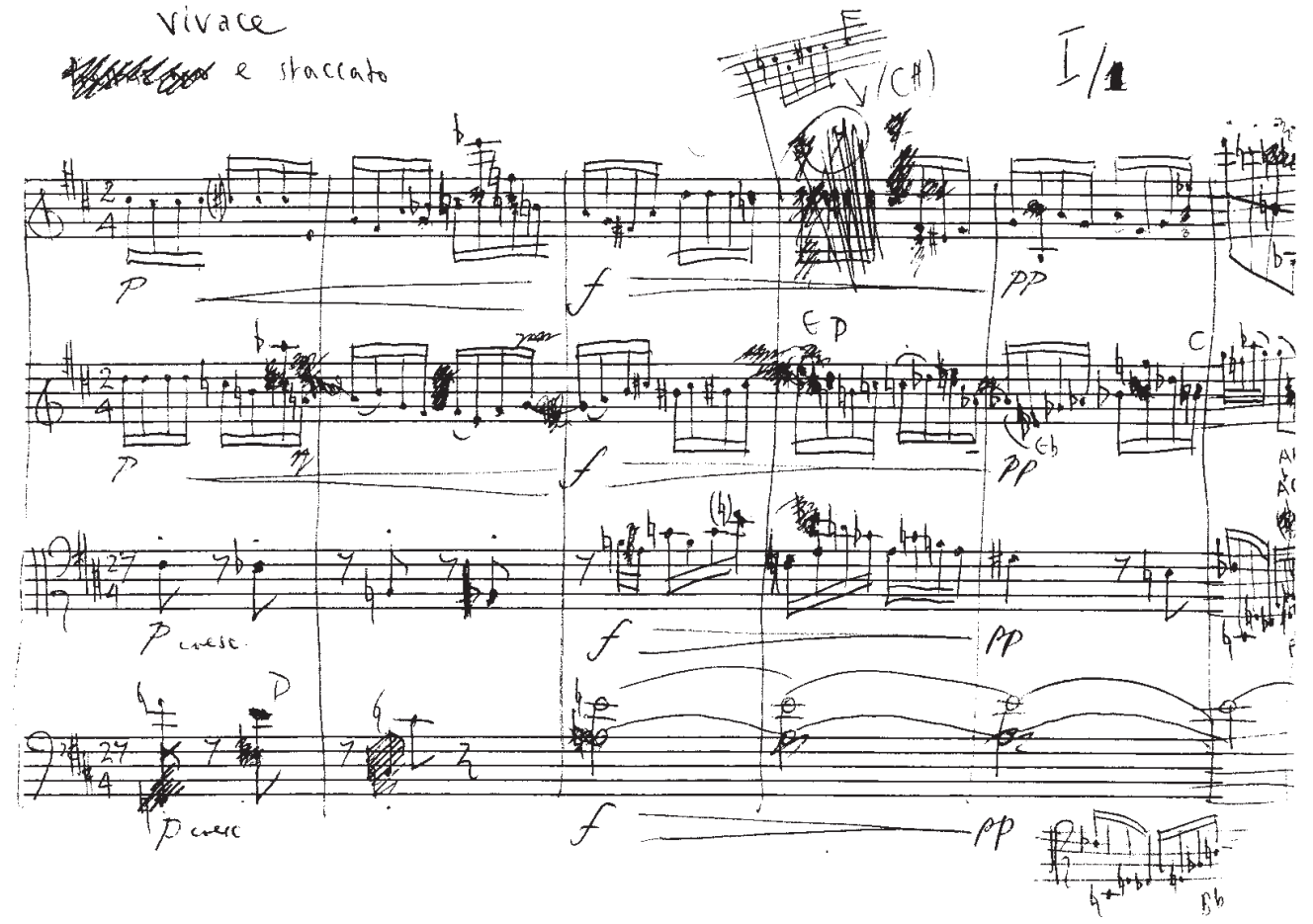

Fig. 1b : Esquisse de la section vivace (mesures 5 à 10) du Deuxième quatuor à cordes de Michael Finnissy 9

9. Reproduit avec l'autorisation du compositeur. Dans l'ensemble de cet article, les citations d'esquisses ou de manuscrits sont la propriété de Michael Finnissy et sont reproduits avec son autorisation. 


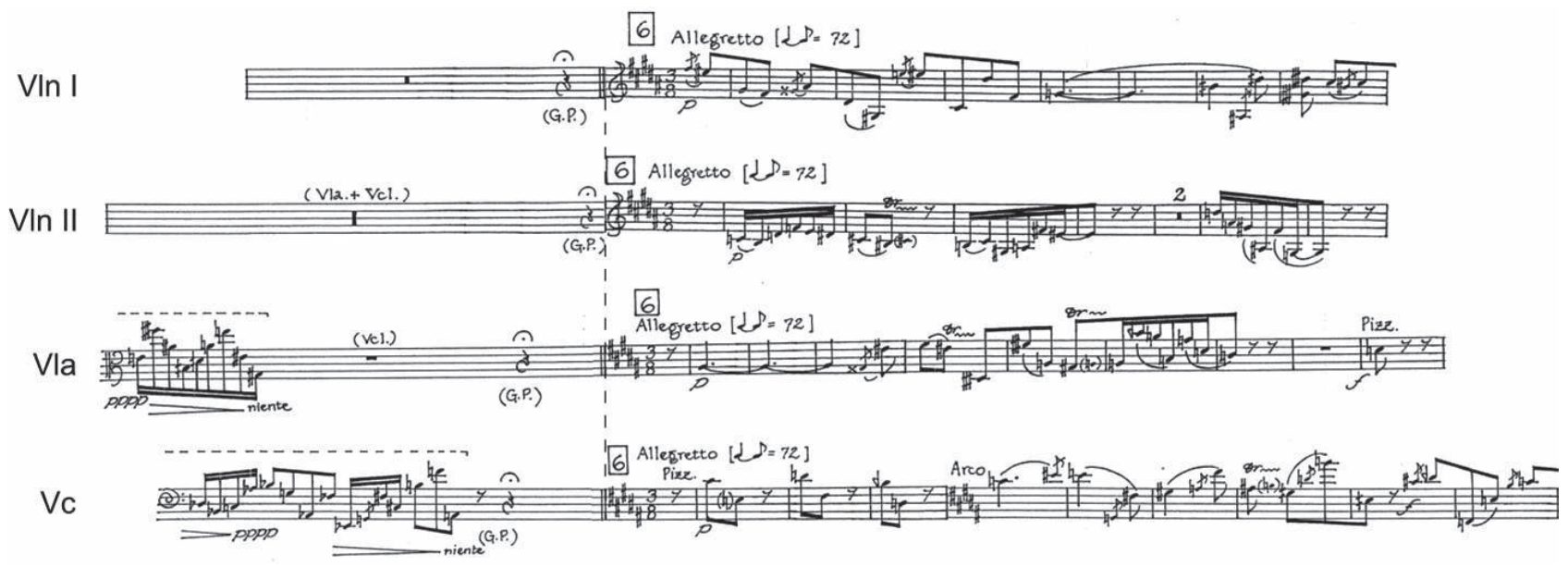

Fig. 2 : Premier système de la section menuet

Finnissy reconnaît qu'il peut avoir subi des influences indirectes de la part d'autres compositeurs de quatuors, car il s'en sert comme modèles auprès de ses étudiants :

Je parle souvent des quatuors à cordes car ils sont un excellent moyen d'aborder la question de la structure musicale. En effet, chez beaucoup de compositeurs, c'est souvent dans le quatuor que le travail sur les structures musicales s'illustre de la manière la plus intéressante.

J'ai ce son dans l'oreille [...]. Alors, bien sûr, on se dit «Tiens, voilà une manière intéressante de réaliser un accord » ou encore « Voilà qui ouvre des possibilités intéressantes ». Mais, avant tout, si je m'en suis tenu à Haydn, c'est parce que cette écriture est tellement différente de celle de mes autres quatuors. Cette pièce est très différente du Premier Quatuor à cordes, en particulier ${ }^{10}$.

En réponse à une version modifiée de la question précédente : «Comment avez-vous résolu le problème posé par la combinaison de matériau né de votre imagination et de matériau externe »? Finnissy répond :

Eh bien, comme vous allez l'entendre quand nous le répéterons, l'œuvre de Haydn n'est pas citée de manière littérale. Ce qui est repris, c'est une espèce de profil rythmique et textural de son quatuor. On le retrouve dans la forme du тепиеt, par exemple, et dans celle de l'adagio. Les contours rythmiques sont conservés, mais pas les hauteurs. Et donc, d'une manière très générale, cela rappelle un quatuor à cordes classique, parce que c'est la tradition à l'intérieur de laquelle j'ai travaillé plutôt que d'essayer d'en faire quelque chose de plus révolutionnaire, ce que j'avais probablement essayé de faire avec mes quatuors précédents. J'ai eu beaucoup de plaisir à dialoguer avec Haydn ${ }^{11}$.

La figure 2 présente des voix individuelles, non alignées verticalement. Finnissy n'a pas réalisé une partition complète car, pour une grande partie de son travail, il n'y a pas de synchronisation définie entre les voix. Même si, dans le menuet, les parties sont rythmiquement synchrones, dans d'autres sections de l'œuvre, au contraire, la coïncidence rythmique doit être évitée et le compositeur a pensé qu'il serait

[...] plus facile d'arriver à ce résultat sans partition - sans en faire une exigence absolue...

Cela semblait pertinent, quoi qu'il arrive, car certains fragments de la pièce allaient être impossible à noter sous forme de partition (les passages où le quatuor se divise en deux duos qui avancent chacun de leur côté). Par conséquent, il allait y avoir des vides dans la partition, pour lesquels il allait falloir adopter un format différent.

10. Entretien avec l'auteure, le 4 février 2007.

11. Ibid. 
Il n'est pas indifférent de présenter quelque chose sous forme de partition complète ou comme un ensemble de parties séparées. S'y joue l'idée que c'est une affaire de polyphonie individuelle, la responsabilité de chacun d'emmener une ligne mélodique vers un point donné avec un phrasé affranchi de la référence aux barres de mesure 12 .

Cependant, ces commentaires n'expliquent pas pourquoi certaines sections de la pièce apparaissent sous forme de partition dans les esquisses (comme dans la figure 1b). À propos de sa manière de travailler, Finnissy précise :

Si j'ai écrit les esquisses sous forme de conducteur, c'est parce que j'avais envie de voir comment les notes s'alignaient. Cela dépend du mouvement d'ensemble de l'harmonie. C'est aussi parce qu'il s'agissait d'une sorte d'expérience néoclassique à la manière de Haydn, ou quelque chose dans le genre. Il semblait que l'harmonie progressait en blocs rapides, à une vitesse à laquelle je n'étais pas habitué. Du coup, je n'étais pas certain que mon oreille, seule, pourrait suffire à la tâche. C'est pourquoi il a fallu que je procède ainsi. On voit bien, sur la partition, qu'elle n'est pas spécialement contrainte par les barres de mesures. Je crois que son utilité se limite à montrer à quoi ressemble le mouvement des textures, autant que celui des harmonies.

Pour moi qui ne joue pas d'un instrument à cordes, ma connaissance des quatuors de Haydn est forcément basée sur la partition. Du coup, pour reproduire «l'effet Haydn », je suis amené à écrire une partition complète. J'estime à $35 \%$ la portion de l'œuvre qui n'est pas notable au format conducteur13.

À propos du lien avec Haydn, Finnissy a fourni aux interprètes le contexte et les explications qui suivent :

Il y a deux choses en parallèle [...] qui ne se marient peut-être pas tout à fait [...]. On retrouve un peu ça dans les quelques travaux néoclassiques de Schönberg et de Berg qui utilisent le même procédé. Ils adoptent le cadre gestuel de la musique classique et y ajoutent des hauteurs chromatiques atonales. PSS : Comme dans la Fantaisie de Schönberg ?

MF : Oui, mais encore plus dans le troisième quatuor. Et, dans une certaine mesure, on retrouve un peu ça dans la Suite lyrique [de Berg]. Donc, on a un certain nombre d'archétypes romantiques, dans le domaine du geste et de la texture, mais les hauteurs ne sont pas les bonnes [...]. Donc, en réalité, il ne faut pas que ça sonne comme si les hauteurs n'étaient pas, disons, « étranges ». Il y a des cadences, enfin, jusqu'à un certain point. Mais il y a quelque chose qui cloche dans les hauteurs. Donc, ça doit sonner comme si les hauteurs ne collaient pas. Les gestes et les stéréotypes classiques, quand on leur enlève la tonalité, on leur enlève tout ${ }^{14}$.

En enregistrant les dialogues et les interactions entre les compositeurs et les interprètes, on fournit aux futurs exécutants un contexte qui va leur permettre de comprendre ce que le compositeur a fait et pourquoi il l'a fait. Ce contexte se révélerait encore plus nécessaire si la situation était différente, notamment si les exécutants n'avaient encore jamais travaillé avec ce compositeurlà. Les membres du Kreutzer Quartet, qui ont déjà joué les œuvres antérieures de Finnissy, se soucient d'établir avec lui un dialogue soutenu. Au cours des répétitions, Finnissy leur a expliqué qu'il avait adopté le cadre gestuel de la musique classique mais qu'il avait aussi emprunté d'autres techniques « gestuelles », par exemple chez Pierre Boulez. Les commentaires du compositeur sur Boulez ont été suscités par une question du violoncelliste, Neil Heyde, pendant une répétition. « La partie qui commence au chiffre 3, quand je joue avec Morgan [l'altiste], [...] on a du mal à donner un sentiment d'agitation. Pourtant, tu as écrit "agité" » (comme on le voit dans la figure 3a, les indications du compositeur à cet endroit sont «senza tempo: irregular and jumpy »). Finnissy fait la réponse suivante :

Vous pourriez, en quelque sorte, exagérer les espaces entre les notes [...]. Dans mon esprit, il ne s'agit pas d'appliquer cette indication à la lettre... Donc je crois que vous pourriez... jouer un peu avec les espaces. En fait, la partie de violoncelle est le rétrograde inverse de la deuxième section du Livre pour quatuor de Boulez [voir fig. 3b], avec les hauteurs du deuxième mouvement de Haydn 15 .

12. Entretien avec l'auteure, le 8 mai 2008.

13. Ibid. Remarque : un « conducteur» est un format de partition où les différentes parties d'un ensemble figurent de façon synoptique (c'est typiquement le cas de la partition du chef d'orchestre par différence avec les « parties séparées » dont disposent les instrumentistes dans l'orchestre).

14. Répétition du 4 février 2007 (PSS correspond à l'intervention du premier violon, Peter Sheppard Skærved).

15. Ibid. 

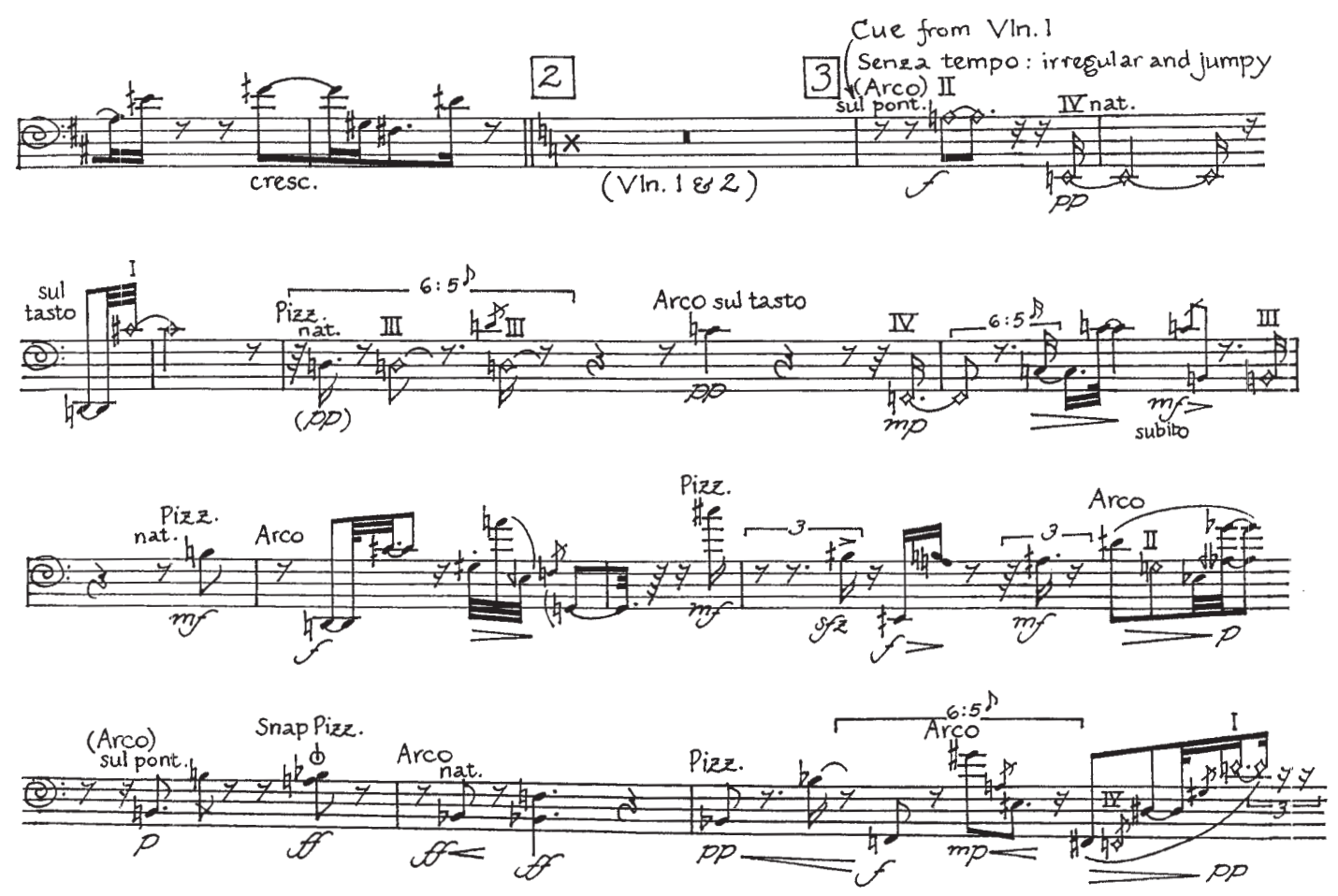

Fig. 3a : Partie de violoncelle à partir du chiffre 3

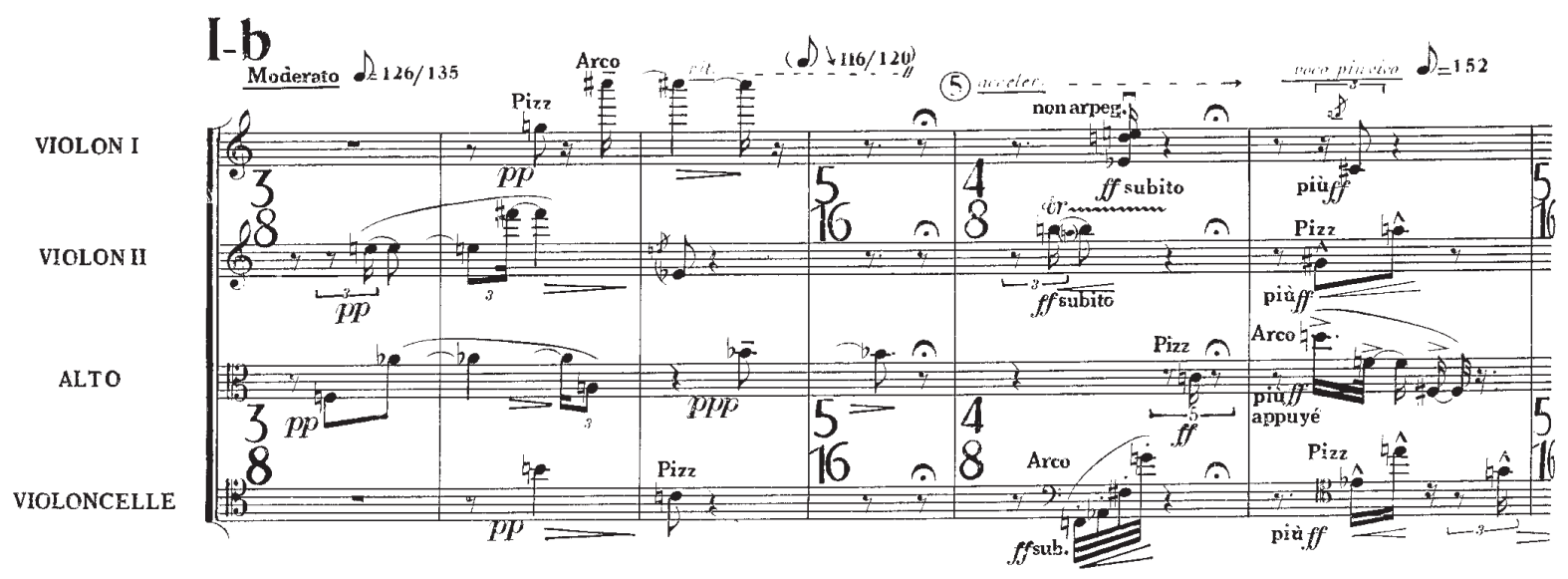

Fig. 3b : Extrait du Livre pour quatuor de Boulez, mouvement I-b (@ Universal Edition, Vienne) 


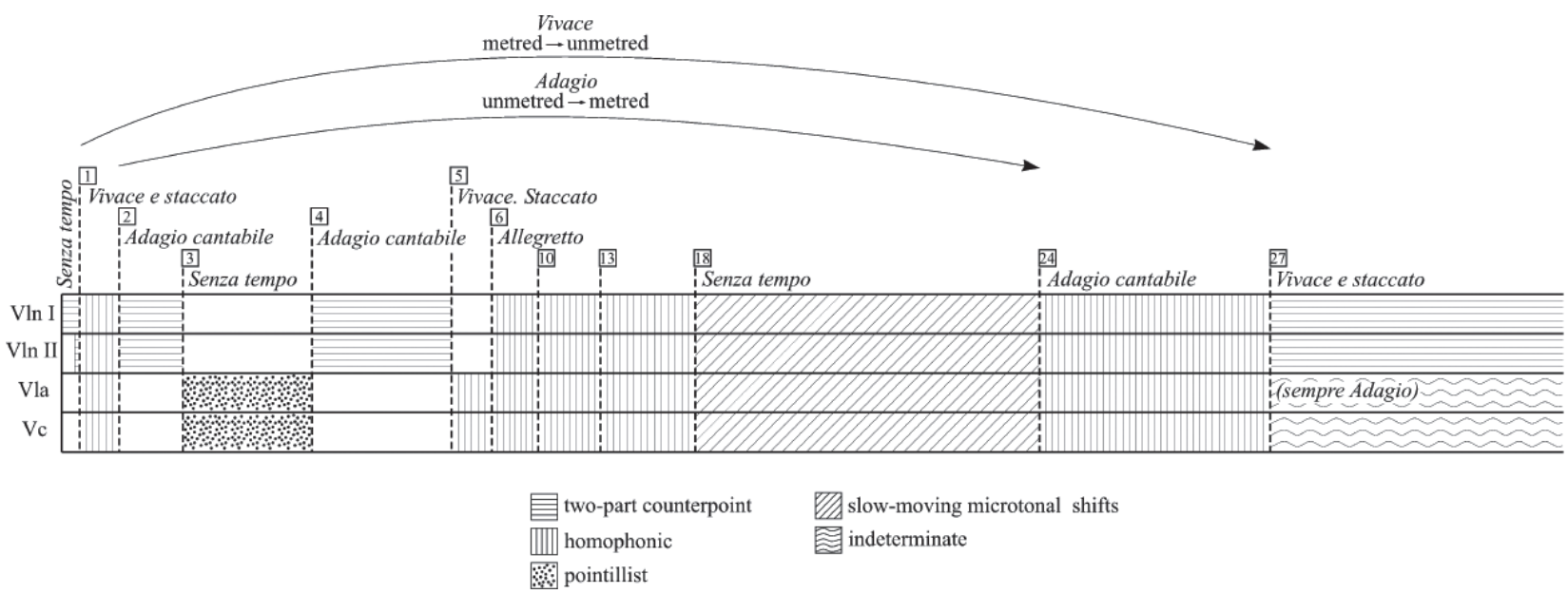

Fig. 4 : Structure texturale du Deuxième quatuor à cordes de Finnissy

Interrogé plus tard sur cet aspect, Finnissy a clarifié sa remarque : c'est la texture de l'œuvre de Boulez qui l'avait attiré. Après une réflexion sur la partition de Boulez, Finnissy a expliqué ce qu'il lui avait emprunté :

Ce n'est que le rétrograde des rythmes. Rien de vraiment littéral [...]. Je crois qu'il y a quelques adaptations : pizzicato..., appoggiatures...

Je voulais quelque chose d'atténué, de nerveux - il y a aussi un brin de satire à l'égard du monde de Darmstadt, qui est si différent de Haydn. Plutôt que de l'évoquer d'une autre manière, j'ai repris littéralement des fragments de matériau [...]. Tout est dans ce va-et-vient accéléré, qui engendre une espèce de monde sonore, même si [...] ça ne sonne pas comme Boulez. Ce n'est pas conçu pour sonner comme Boulez. Ce n'est que par moments que l'on retrouve cet effet de kaléidoscope, qui ne ressemble à rien d'autre dans la pièce ${ }^{16}$.

Le diagramme de la figure 4 propose une vue d'ensemble des rapports entre plusieurs sections, de styles gestuels et de textures opposés. La pièce y est segmentée en fonction de la texture. Ce diagramme, résultat d'un travail d'analyse plutôt que représentation d'un processus précompositionnel, peut aider le lecteur/auditeur à s'orienter à l'intérieur du morceau.

Cette représentation de la texture a été dessinée de manière proportionnelle, en fonction des minutages de la création mondiale, qui a eu lieu le 13 février 2007. Si ce diagramme délimite bien les différentes sections, il ne montre pas pour autant les transitions ou les différents types d'enchaînement qui existent entre les sections et qui sont décrits plus bas. Un peu partout dans la pièce, il y a des liens significatifs entre le tempo et la texture qui méritent d'être explorés. Par exemple, le premier et le deuxième vivace ont une texture verticale, alors que le dernier vivace est écrit comme un contrepoint à deux voix. Inversement, les deux premiers adagios sont écrits en forme de contrepoint à deux voix tandis que le troisième adagio, de son côté, est écrit verticalement. Ces changements de texture vont être réexaminés à la lumière des méthodes de travail de Finnissy.

\section{Méthodes de travail}

Lors de notre premier entretien avec Finnissy, nous l'avons d'abord interrogé sur la façon de travailler son matériau, et sur le rapport général entre matériau et processus, avant de recentrer la question sur l'œuvre considérée. Il nous disait ainsi, avant d'en commencer l'écriture :

16. Entretien avec l'auteure, le 8 mai 2008. 
[...] quelque part, le matériau, ce sera [...] des notes et des rythmes. Mais ces notes et ces rythmes émanent-ils de l'idée de quatuor ou de quelque chose d'autre que j' aurais envie de faire ? Par exemple, de répétitions ou d'une technique particulière de variations ou bien d'autre chose encore? Dans ce cas, la technique de variation prend la place du matériau, et cesse d'être un élément spécifique [...]. Dissocier le processus de ça $[\ldots]$. Et puis $[\ldots]$ il y a cette idée, que certains considèrent comme caractéristique de la pensée moderniste : on attend de l'auditeur qu'il repère ou qu'il perçoive le processus à travers l'écoute, comme une partie intégrante de l'écoute. Je ne suis pas certain d'isoler le processus, à la manière d'un Steve Reich, par exemple, parce que, d'autre part, [...] enfin, pas vraiment maintenant, mais plus tôt, dans beaucoup de mes anciennes pièces (par exemple Nobody's Jig) [...] il n'y pas vraiment un processus qui serait imposé au matériau. C'est une anthologie de fragments de matériau et il y a une certaine forme de correspondance d'une partie à l'autre ; leurs sources se recouvrent partiellement. Mais je ne sais pas si on peut vraiment décrire ça comme un processus - au sens d'un travail effectué sur quelque chose - parce que la somme de travail est minimale. C'est assez proche des assemblages. J'aime bien les assemblages ${ }^{17}$.

Une fois l'écriture du quatuor terminée, Finnissy a été interrogé sur cette affirmation selon laquelle il travaillerait souvent en créant des assemblages (au lieu de « simuler une espèce de processus organique $18 \gg)$. Quand on lui demande si c'est bien l'approche qu'il a adoptée pour cette pièce, il répond :

Oui et non. [...] En général, je vois la composition comme un moyen de placer des choses différentes dans un espace commun. Et je n'ai jamais souscrit aux dogmes du sérialisme ni du diatonisme, ni à aucun «isme », ni à la nouvelle complexité. [...] Donc, j'aime bien que les choses aient leurs contraires au sein du même espace. Comme le blanc et le noir, le doux, le complexe, le simple. Et dans le cas qui nous occupe, comme j'étais tombé sur ce quatuor de Haydn et que j'avais décidé de l'utiliser, naturellement, j'ai voulu travailler aussi avec un matériau qui soit [...] une sorte d'anti-Haydn. Nous ne sommes plus au XVIII ${ }^{\mathrm{e}}$ siècle, nous sommes en 2008 [...]. Et si le classicisme a un sens quelconque, c'est en tant que point de référence. C'est un classicisme que nous percevons d'une manière très différente de Haydn. Parce que, naturellement, c'était de la musique contemporaine quand Haydn l'a écrite [...]. Je ne tenais pas tellement, à travers mon écriture, à entrer dans ce genre de débat. Donc, dans une certaine mesure, ma pièce « assemble » ces éléments 19 .

Dans l'interview de février 2007, juste avant les premières répétitions, Finnissy est allé un peu plus loin sur la manière de passer d'un type de matériau à un autre :

Quand on voit le matériau, on voit quels fragments présentent tel type de continuité et lesquels sont manifestement le produit d'un montage par contraste avec un matériau de type différent. Un peu comme on perçoit visuellement la différence entre une cassure brutale - on passe brusquement d'un plan à un autre - et une transition lente où une image se fond dans une autre. Ces deux types d'enchaînement ont un effet émotionnel et psychologique différent. Dans cette pièce, je me suis servi des deux 20 .

La cassure brutale d'un plan à un autre est manifeste au début de la section Menuet. À la fin de la section Vivace, à partir du chiffre 5 , les exécutants se rejoignent pour une pause générale juste avant le début du menuet (voir la figure 2, supra). Un exemple de transition lente, où une image se fond dans la suivante, s'observe à partir du chiffre 1 jusqu'après le chiffre 2. À la fin d'un court tutti comprenant le premier vivace de la pièce, la partie d'alto fait la jonction avec le début du premier duo entre les deux violons ; les trois voix sont représentées dans la figure 5.

En mai 2008, Finnissy développe cette analogie entre sa méthode de travail et les techniques cinématographiques :

C'est comme [...] le montage d'un film. On a vu certains passages, puis d'autres bouts de film pris un peu plus loin, et constitués des mêmes fragments que précédemment, mais découpés en plus petits bouts. Ces morceaux sont rattachés les uns aux autres de diverses manières. Ainsi on retrouve le principe de la réexposition, avec un aperçu accéléré de l'ensemble du matériau, mais pas au sens littéral, si bien que tout est réordonné en permanence [...], une combinaison de [...] matériau fraîchement composé avec des réminiscences de [...] parties de la pièce, diversement transformées ${ }^{21}$.

17. Entretien avec l'auteure, le 10 juillet 2006.

18. Ibid.

19. Entretien avec l'auteure, le 8 mai 2008.

20. Entretien avec l'auteure, le 4 février 2007

21. Entretien avec l'auteure, le 8 mai 2008. 

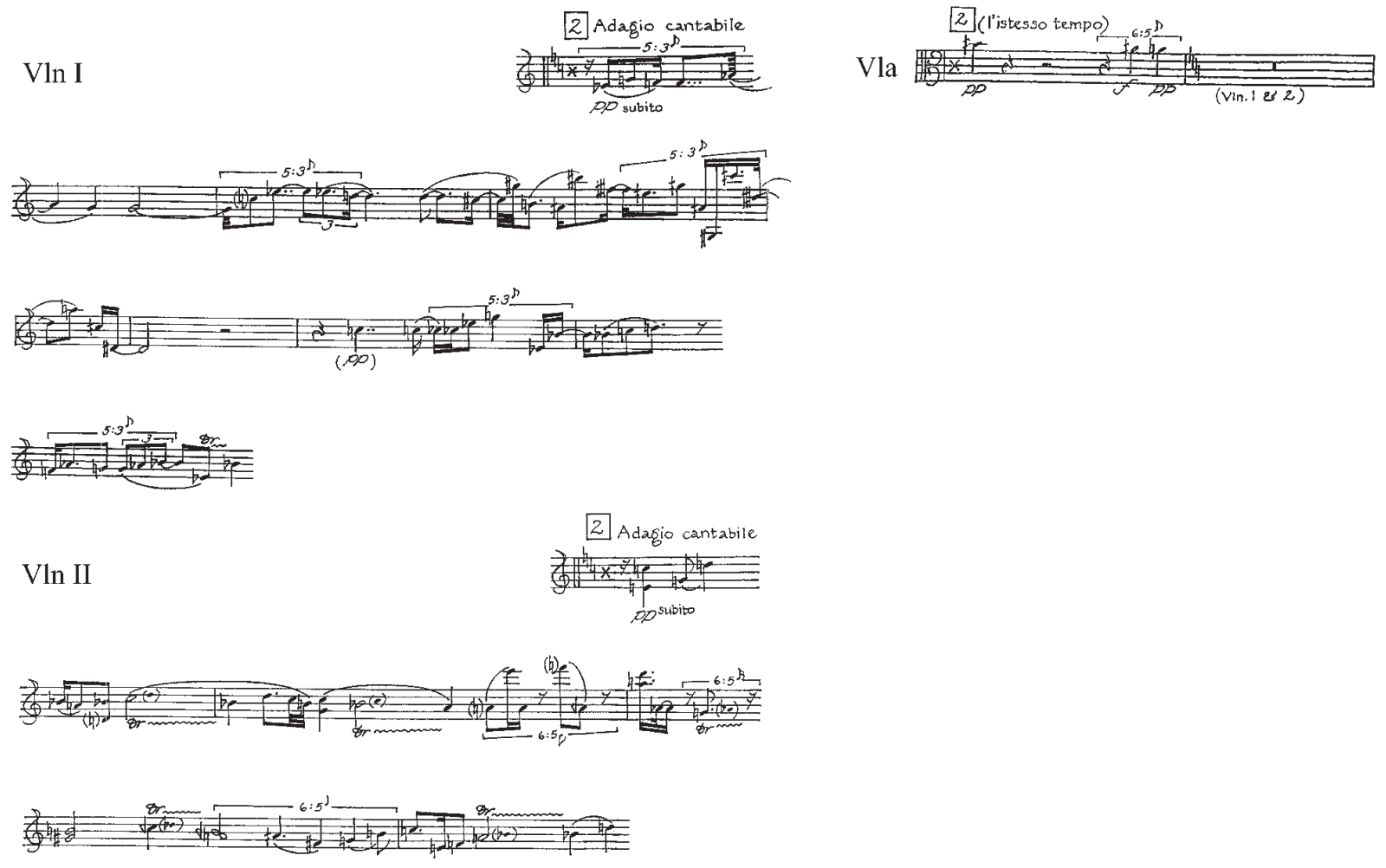

Fig. 5 : Début de 1'Adagio cantabile, au chiffre 2

Finnissy transforme différents types de matériaux, de plusieurs manières. L'une d'elle est la permutation de schémas rythmiques et de hauteurs utilisés ailleurs dans son œuvre, ainsi que cela a déjà été décrit dans un entretien avec le compositeur paru en 1997 :

Dans le cas d'un matériau né de mon imagination, il m'arrive d'écrire séparément les configurations de rythmes et de hauteurs, puis de les combiner pour voir ce que ça donne. Il $\mathrm{y}$ a toujours moyen de les déplacer et de faire quelque chose d'autre si ça ne marche pas, et le matériau d'origine continue d'exister. C'est une manière passionnante de composer car tout devient une aventure 22 .

Parmi les esquisses du Deuxième quatuor, deux pages présentent une liste de trente cellules rythmiques différentes et deux autres pages présentent une liste de cinquante cellules mélodiques. Les figures $6 \mathrm{a}$ et $6 \mathrm{~b}$ sont extraites de ces deux listes. Finnissy compose avec ces cellules rythmiques et mélodiques, dans un ordre qui lui est dicté par une liste aléatoire de nombres qui, comme il l'expliquait déjà, provient de la source suivante :

J'ai un document que j'ai imprimé et relié, appelé « Tableaux de nombre aléatoires », de Lincoln E. Moses et Robert V. Oakford, publié en 1963. Il contient [...] des permutations aléatoires de chiffres en nombre limité : 1-9, 1-16, 1-20, 1-30, $1-50,1-100,1-500,1-1000^{23}$.

22. «Conversations with Michael Finnissy », dans Uncommon Ground. The Music of Michael Finnissy, dir. Henrietta Brougham, Christopher Fox et Ian Pace, Aldershot, Ashgate, 1997, p. 40.

23. Ibid. 
(1) $1 \sum^{m f}=\square^{2}$

(2) $7 \sum_{p p} \sum_{m f} \cdots L_{m f}^{p}$

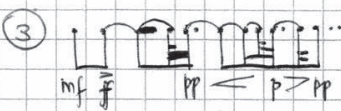

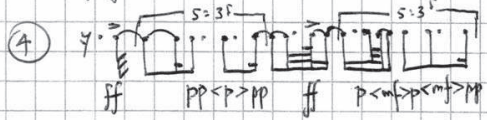

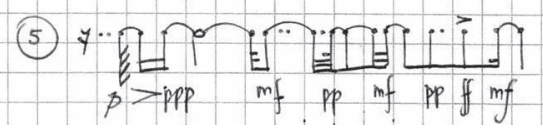

$9.6 \cdot 27 \cdot 31 \cdot 30 \cdot 18 \cdot 13 \cdot 19 \cdot 10 \cdot 28,18,72$
$+14 \cdot 7 \cdot 23 \cdot 4 \cdot 8 \cdot 8 \cdot 12 \cdot 24 \cdot 17,1,20 \times 16$

22. $25 \cdot 3 \cdot 26$.

Fig. 6a : Liste de cellules rythmiques extraite des esquisses du Deuxième quatuor à cordes de Finnissy

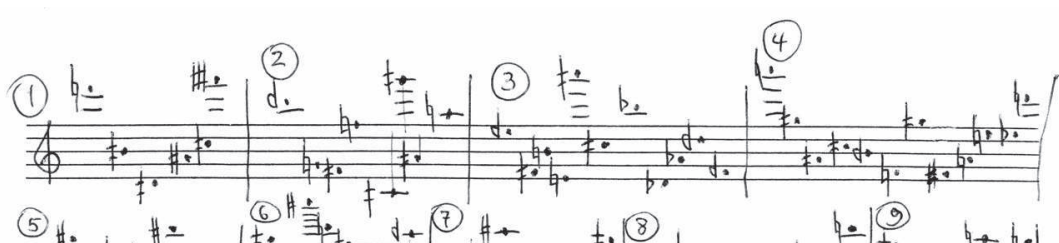

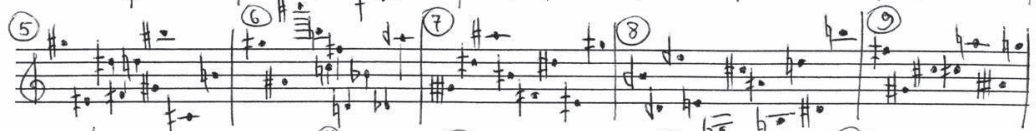

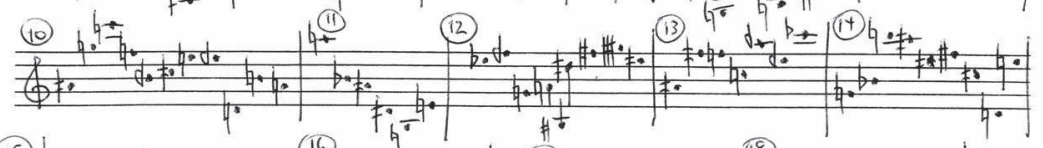

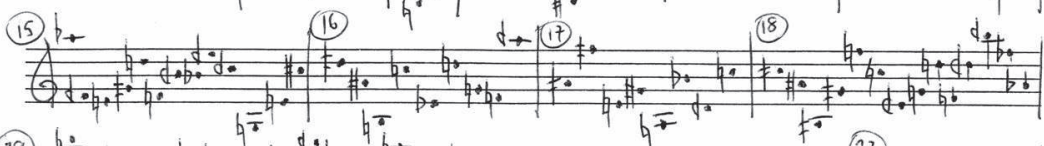

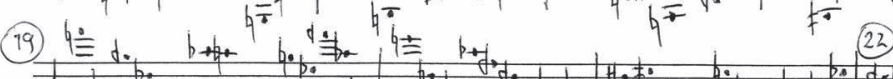

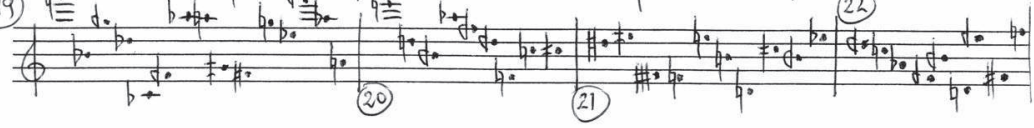

(23)
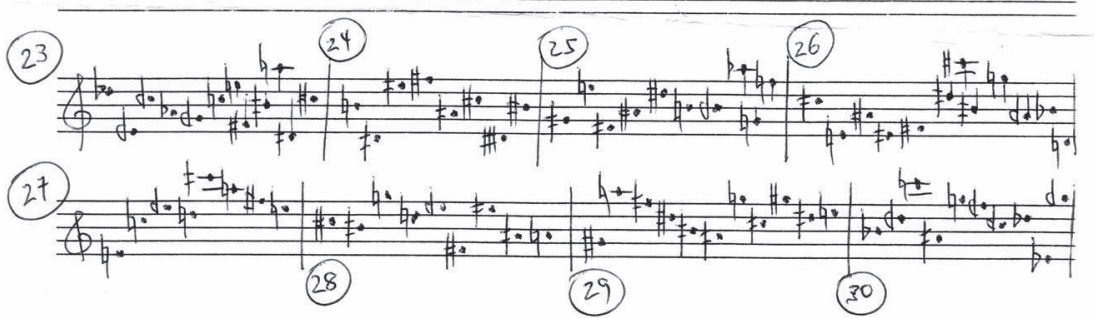

Fig. $6 \mathrm{~b}$ : Liste de cellules mélodiques extraite des esquisses du Deuxième quatuor à cordes de Finnissy 
Finnissy explique comment il intervertit ces matériaux : «Je procède de la manière suivante [...] quand j'utilise les nombres aléatoires. Je note les plages disponibles puis j'étiquette les notes et je les range dans un ordre aléatoire 24 . » Les nombres qui figurent en haut à droite figure $6 \mathrm{a}$ montrent que le compositeur barre chaque permutation une fois qu'il l'a utilisée. Cette organisation des cellules rythmiques s'observe dans la partie de premier violon du premier adagio (voir fig. 5). En revanche, les signes de nuances et d'accentuation présents dans ces permutations ne sont pas transférés dans l'esquisse des parties ou dans les parties définitives, ce qui dénote l'existence d'un facteur de choix, plutôt qu'une adhésion stricte au schéma de permutation. De surcroît, des silences ont été ajoutés à la permutation 27, et les rythmes irrationnels 11:7 et 9:7 ont été changés en 5:3. Voici ce que dit Finnissy de la liste de hauteurs de la figure $6 \mathrm{~b}$ :

[...] un réservoir de hauteurs utilisé encore et encore, avec sans doute très peu de variations, si ce n'est quelques octaviations $[. .$.$] . Ça ressemble beaucoup à un [. .$.$] prolongement$ d'une sorte de Grundgestalt, ou de technique des douze sons dans laquelle le matériau utilise un réservoir limité, selon un schéma défini. Cela dit, c'est beaucoup plus large qu'une série car une série ne duplique pas les mêmes motifs. Mais on les retrouve dans toutes les parties, en tant que matériau, de temps en temps. Donc, théoriquement [...] ils confèrent une certaine unité au matériau. En même temps on [...] ne le remarque pas dans la pièce parce que l'archétype de tel ou tel type de matériau n'est pas produit par la répétition thématique. Il émane plutôt des répétitions texturales ou gestuelles. Ou des éléments rythmiques, peut-être.

On trouvera ce type de motifs vers le début, tandis que la musique plus tonale s'appuie sur des quasi-citations ou des passages issus de Haydn; parce qu'ici [montrant la figure 6b], apparemment, on est plutôt dans le quart de ton et on y retourne, dans les duos pour violons à la fin 25 .

Qu'il s'agisse du matériau rythmique ou des hauteurs, Finnissy explique que les permutations ne sont qu'un « moyen de produire des variations du matériau avec des ordonnances aléatoires des éléments constitutifs, de manière à ce que les interprètes ne les retrouvent pas dans le même motif 26 ». Certaines pages des esquisses présentent également une compression du matériau chromatique en quarts de tons et, dans d'autres pages qui concernent la section Senza tempo, à partir du chiffre 18, en huitièmes de tons.

Une autre méthode de transformation se révèle à travers la manière dont Finnissy a travaillé avec le matériau de Haydn :

[...] l'autre élément du quatuor de Haydn qui s'est introduit dans le mien, c'est son écriture très organique. Donc, j'ai vraiment pensé à traiter le matériau [...]. Je pense qu'on dirait couramment «à le développer » mais je préfère dire «l'explorer ». C'est vrai, j'ai varié le matériau et je l'ai travaillé d'une manière qui est, $\mathrm{j}$ 'imagine, plus proche d'un processus compositionnel organique que d'un collage pur et simple. Il y a $[\ldots]$ un peu des deux 27 .

Ce processus compositionnel « organique » apparaît clairement dans les sections Adagio de la pièce (voir fig. 4), avec la combinaison et la recombinaison de fragments motiviques issus de l'Adagio de Haydn, op. 64 $\mathrm{n}^{\circ} 5$. Cependant, il faut d'abord évoquer la version écrite par Finnissy du premier thème de violon de Haydn (fig. 7a). Celui-ci n'apparaît qu'au troisième adagio. Il est joué par l'alto au chiffre 24 (fig. 7b). Au début, le premier violon reprend les hauteurs qui étaient celles de l'alto dans la version de Haydn. Puis c'est lui qui récupère la mélodie au chiffre 26. Toutes les parties sont transposées et les deux parties de violon sont également l'objet de variations sur le plan rythmique.

Finnissy explore des éléments éclatés du matériau motivique, dans les deux premiers adagios (aux chiffres 2, puis 4) avant la véritable exposition du thème qui n'intervient qu'au troisième adagio. La figure 8 identifie quelques-unes des cellules présentes dans les deux parties de violon du premier adagio, parmi celles qui ont des correspondances intervalliques et rythmiques avec l'Adagio de Haydn'28.

24. Entretien avec l'auteure, le 8 mai 2008.

25. Ibid.

26. Ibid.

27. Ibid.

28. Pour une analyse détaillée des rapports entre les motifs des différents adagios, voir A. Bayley et M. Clarke, art. cit. 

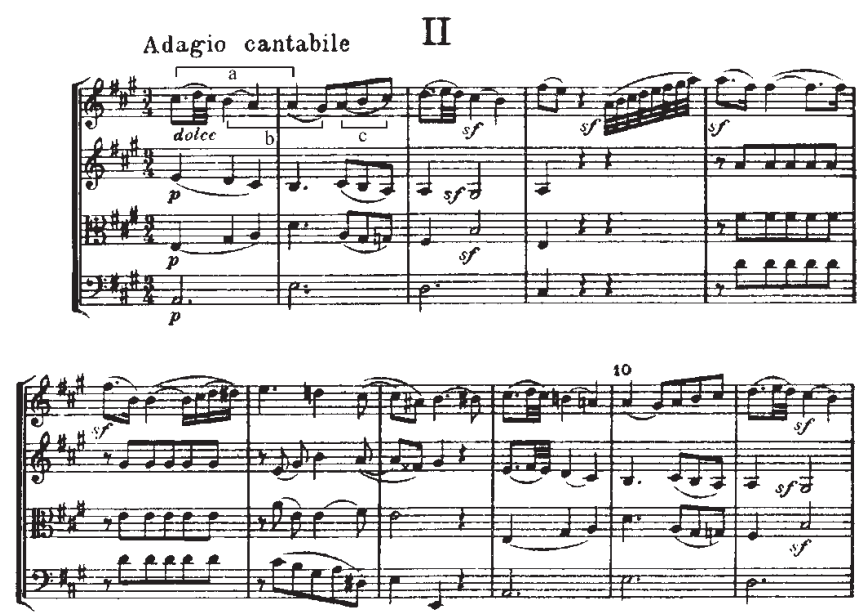

Fig. 7a : Haydn, op. $64 n^{\circ} 5$, ouverture du deuxième mouvement, Adagio cantabile

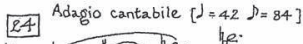

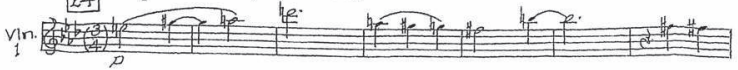

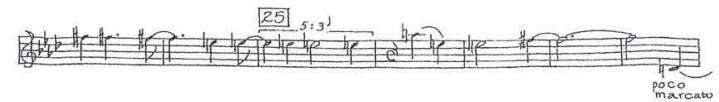

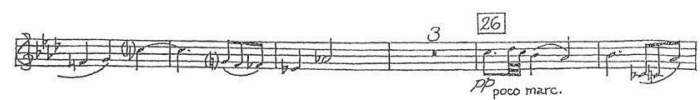

14.0.

24 Adagio cantzbile $[J=42 \quad \rho=84.1$

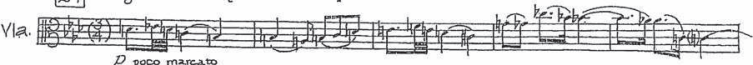

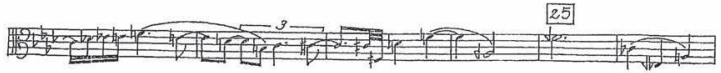

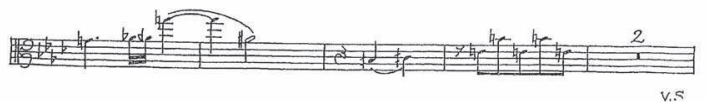

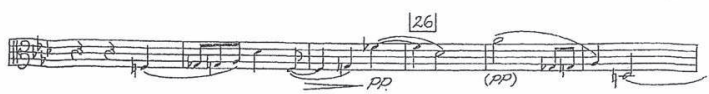

$[24]$ Adagio cantabile $[J=42 \quad D=84]$

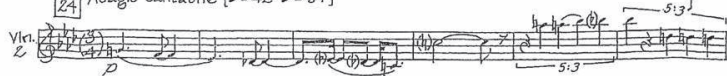
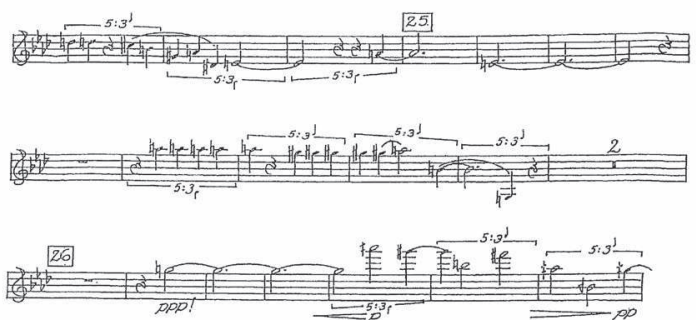

24 Adagio cantabile $[J=42 \quad D=84$

var \$

Poc marcato

2

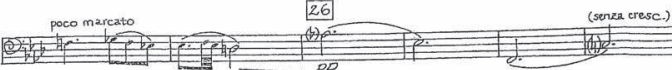

Fig. 7b : Deuxième quatuor à cordes de Finnissy, troisième section Adagio cantabile 

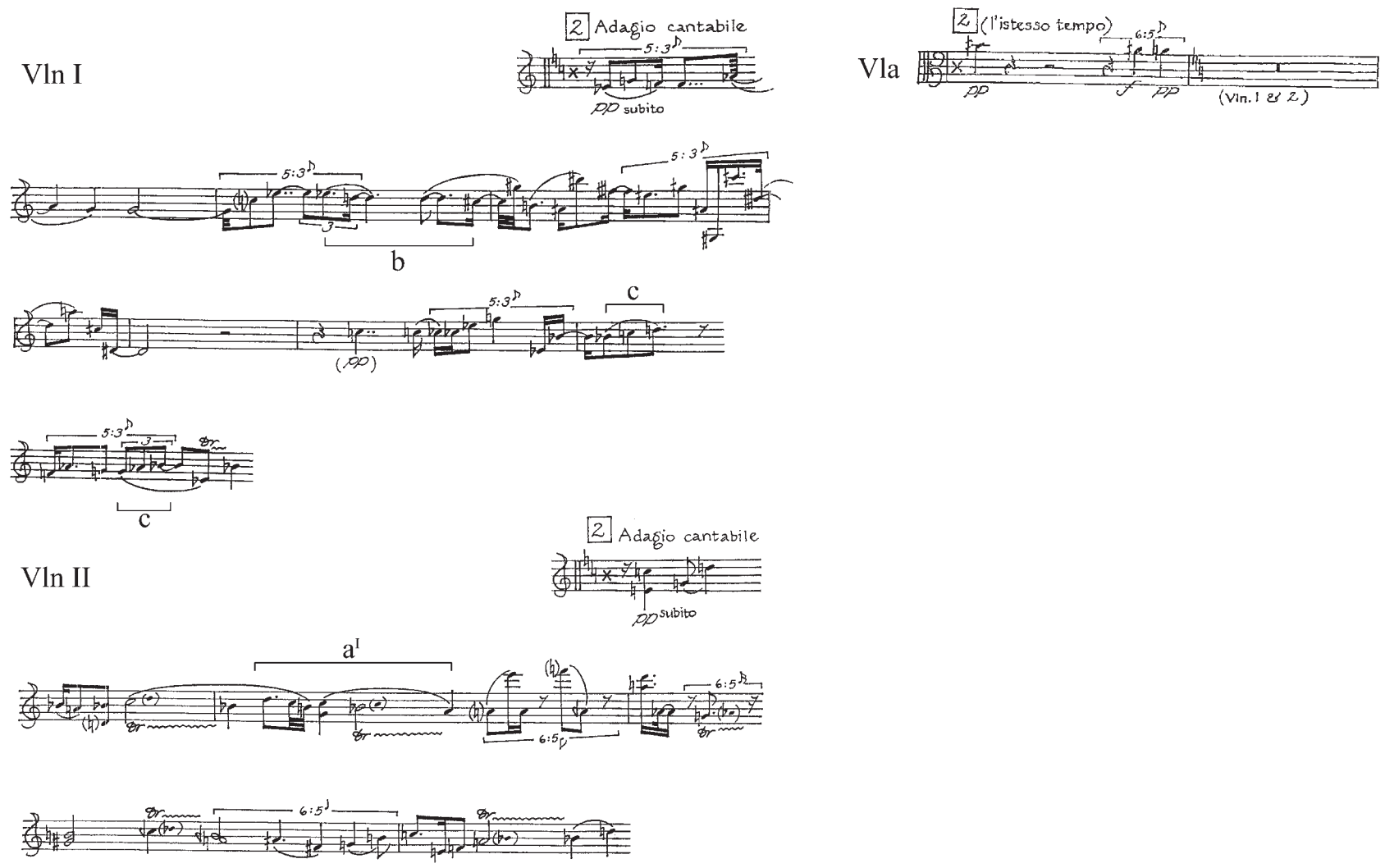

Fig. 8 : Deuxième quatuor à cordes de Finnissy. Adagio cantabile, chiffre 2

Sa manière d'ordonner et de structurer le matériau détermine la conception des sections Adagio, qui évoluent d'une version non mesurée vers une version mesurée (voir fig. 4, supra). Interrogé à ce sujet, Finnissy répond ce qui suit :

J'imagine toujours que le matériau fait une sorte de voyage. Quelquefois, ce voyage est préparé, d'autres fois il résulte de mon travail sur le matériau. Je ne prévois pas nécessairement qu'il va s'agir, comme ici, d'un voyage du non mesuré vers le mesuré. Cela va découler de mon travail sur le matériau. Et l'on va presque toujours d'un point à un autre. Je ne dirai pas « du début jusqu'à la fin », car cela impliquerait qu'il y a un point de départ et un point d'arrêt. Or, j'ai tendance à éliminer ces notions car, dans mon esprit, la musique existe, les compositions existent, à l'intérieur d'un continuum, dont le début est une espèce d'illusion qui naît avant le concert et dont la fin advient après que le public est parti. Néanmoins le matériau voyage au cours de la pièce, donc, nous sommes probablement dans une démarche de ce genre 29 .

Quand on fait remarquer à Finnissy que les sections Vivace semblent contredire cette idée, puisqu'elles commencent de manière mesurée pour devenir non mesurées au chiffre 27 (voir fig. 4), il répond :

Et bien, encore une fois, si je m'en suis rendu compte et si j'ai pris ce genre de décision, c'est certainement que j'aime travailler sur ce genre d'hybridations. Il est assez rare que tous les types de matériaux présents dans une pièce fassent le même voyage. Ils vont probablement plus souvent dans des

29. Entretien avec l'auteure, le 26 septembre 2008. 
directions opposées que dans la même direction [...]. Il y a un élan fondamental que je dois mettre en place, des sortes de conflits entre les matériaux que je puisse ensuite résoudre, mais la résolution installe un autre conflit qui nécessite une nouvelle résolution et ainsi de suite ${ }^{30}$.

Interrogé précisément sur ses structures harmoniques, Finnissy explique :

En réalité, mon sens harmonique est bâti sur des progressions à base de médiante et de sus-dominante. J'ai appris à les connaître et à les aimer à travers Beethoven et Schubert. Du coup, les tonalités ont tendance à progresser par tierce, ascendante ou descendante 31 . Il y a un autre type de progression que j'affectionne particulièrement, c'est le napolitain. On s'élève d'un demi-ton. Quant à savoir si cela est conçu comme quelque chose de fonctionnellement tonal, je suis convaincu que ce n'est pas vraiment le cas. J'ai tendance à pratiquer ce genre de choses sur le court terme plutôt que sur le long terme ${ }^{32}$.

Il est intéressant de noter que c'est cette relation semitonale que Finnissy a associée au matériau de l'Adagio de Haydn, l'original étant en la majeur, tandis que la version de Finnissy est théoriquement en la bémol majeur (voir fig. 7). Finnissy déclare qu'il utilise les armures « uniquement pour orienter l'exécutant dans une direction particulière ». Il explique que, quelle que soit l'armure,

[...] la musique ne restera pas longtemps dans cette tonalité, sans changements d'altérations. Ce sont des sortes d'emplacements tonals qui permettent à l'interprète de replacer ce qu'il fait dans un cadre, celui de la relation entre deux tonalités. Car, habituellement, cela peut s'appliquer à une tonalité majeure et sa relative mineure. Et ce ne sera pas nécessairement sans ambiguïté. C'est essentiellement ambigu 33 .

Finnissy ne fait pas non plus mystère de son adhésion à la notation enharmonique (pour laquelle toute note altérée peut être exprimée aussi bien en bémol qu'en dièse) :

Pour moi, cela ne fait pas partie d'un mécanisme qui permettrait de reconnaître les tonalités et de les manipuler de manière cohérente. Bien sûr, à un niveau plus superficiel, elles [les armures] pourraient être aussi une simple affirmation concernant l'utilisation de matériaux tonals. Mais comme elles ne sont pas utilisées d'une manière tonale, en tout cas pas dans un sens fonctionnel, là n'est pas leur rôle essentiel. [Au lieu de cela] une armure apporte une idée de relativité, car on va et on vient entre des emplacements qui sont plus ou moins forts ou faibles en fonction des conventions de la structure tonale, et cela rend un grand service, car cela constitue une solution alternative au phrasé, à l'accentuation et aux dynamiques - toutes choses pour lesquelles je me montre un peu réticent. Je n'abuse pas des signes de dynamiques sur les partitions, sinon pour [...] des raisons stylistiques ${ }^{34}$.

En faisant appel à des enharmonies, par exemple, en utilisant des dièses dans le contexte d'une armure à quatre bémols (au cours du troisième adagio), Finnissy met l'accent sur l'importance de reconnaître ce que fait le matériau et comment il fonctionne dans un contexte particulier :

Ça dépend de ce qu'il est censé rappeler. Je peux chercher à remettre en mémoire un passage antérieur dans lequel il y aurait une armure à dièses. Ce rappel, je peux aussi le destiner à l'interprète. Ou encore, il peut y avoir l'idée de mettre votre sens tonal en échec ou simplement de le brider, ce qui, une fois de plus, crée une sorte de tension particulière dans l'exécution, une tension que l'on ne saurait obtenir en utilisant une orthographe atonale non spécifique. J'aime le caractère dense de tout cela 35 .

L'analyse harmonique de cette pièce mériterait, entre autres choses, une recherche plus approfondie, en rapport avec les tensions à l'œuvre pour d'autres paramètres. Même si les remarques du compositeur sont, en tant que telles, riches d'enseignements pour les exécutants, on peut envisager une étape ultérieure qui consisterait à mettre ces remarques en perspective avec la vision des exécutants, telle qu'elle apparaît dans les questionnaires écrits et dans les discussions interactives

30. Ibid.

31. Ces progressions sont indiquées assez clairement dans les esquisses.

32. Entretien avec l'auteure, le 26 septembre 2008.

33. Ibid

34. Ibid. La seule exception qui vienne à l'esprit de Finnissy est la section dans laquelle il parodie Boulez et où, par conséquent, il déploie toute une variété de dynamiques.

35. Entretien avec l'auteure, le 26 septembre 2008. 
que nous n'avons pu commenter ici, faute de place ; la signification psychologique du style notationnel de Finnissy est examinée de manière plus approfondie dans une autre publication 36 .

\section{Conclusion}

Les commentaires présentés ici à propos du Deuxième quatuor à cordes de Finnissy montrent dans quelle mesure une approche ethnographique de la composition peut produire nombre d'informations irremplaçables pour la compréhension d'une œuvre. Le fait de retracer les conceptions du compositeur et de consigner ses interactions avec les interprètes permet de rendre publiques des perspectives nouvelles et de première main. En documentant les points de vue des sujets observés, cette approche offre aux élèvesinterprètes, aux exécutants et aux compositeurs un outil précieux qui leur donne accès aux intentions de l'auteur - ce qui, en principe, pourrait soulever des objections, dans notre ère post-« mort-de-l'auteur ». Cependant, les éléments (inter)subjectifs que cette approche met en avant enrichissent en même temps les ressources plus « objectives » que constituent la notation, les esquisses et l'enregistrement sonore d'une pièce. De telles dimensions subjectives sont généralement occultées par l'analyse musicale traditionnelle. Pourtant, ainsi que la présente étude tente de le démontrer, les remarques des participants peuvent contribuer efficacement aux processus tant d'interprétation que d'analyse et prendre en compte leurs interactions à différentes étapes de la composition et de l'interprétation permet à l'ethnographe de produire toutes sortes de narrations.

Finnissy est un compositeur au discours relativement transparent, qui donne généralement des raisons explicites à ses décisions. Cela peut déformer l'analyse en l'orientant avec force dans telle ou telle direction autorisée. Il est donc important de combiner les différentes perspectives apportées par le compositeur avec les questions et les remarques des interprètes, ainsi que celles de l'auteure du présent article. Ainsi que nous l'avons vu, Finnissy discute de ses méthodes d'écriture en évoquant la notion d'unité créée à partir du matériau.
D'un autre côté, il explique que l'absence de répétition thématique empêche l'auditeur d'avoir conscience d'une quelconque unité. Au lieu de cela, l'attention de ce dernier serait attirée par les qualités gestuelles, texturales ou rythmiques de la musique. À ces remarques perspicaces du compositeur, il convient d'opposer d'autres commentaires qui révèlent une distinction moins claire entre ses décisions conscientes et inconscientes ou, en tout cas, moins évidente que dans sa relation des faits. Par exemple, sa manière de répondre à notre question concernant l'évolution métrique des différents types de matériau suggère que cette évolution fut sans doute une conséquence fortuite plutôt que quelque chose de programmé. Globalement, les avantages procurés par la possibilité d'interroger Finnissy sur ses méthodes de travail compensent largement les quelques occasions où il s'est reconnu incapable de se rappeler ce qu'il avait fait exactement. La référence qu'il fait au montage de film, par exemple, est de nature à intéresser particulièrement les autres compositeurs ; elle serait également utile à approfondir dans un contexte d'analyse musicale. Il faudra une autre étude pour faire une place à toutes les pistes ouvertes par cette recherche.

Les questions sur les esquisses ont conduit le compositeur à expliciter, de manière pertinente et détaillée, ses façons de procéder et sa conception de la pièce. En dehors des listes de hauteurs et de rythmes illustrées figure 6, les esquisses de Finnissy ressemblent d'assez près au résultat final, au moins dans le contenu, sinon dans la forme. Par conséquent, certaines des questions sujettes à controverses et touchant à l'utilité d'examiner les esquisses pour alimenter l'analyse musicale sont, soit sans objet, soit moins problématiques qu'elles ne le sont pour d'autres compositeurs. Pour sa plus grande partie, le matériau figurant dans les esquisses apparaît tel quel dans sa forme mélodique pleinement développée, accompagné, çà et là, par des calculs concernant l'utilisation de modèles mélodiques et rythmiques. La manière dont ces calculs déterminent

36. Amanda Bayley et Neil Heyde, « Communicating through Notation: Michael Finnissy's Second String Quartet from Composition to Performance ", dans Notation and Practice: Essays in Musical Performance and Textuality, dir. Ronald Woodley, Cambridge, Cambridge University Press (à paraître). 
la notation finale est rarement claire, y compris pour Finnissy lui-même, ce qui suggère qu'ils sont plus un guide qu'une règle à laquelle il faudrait obligatoirement adhérer. Cela laisse une place à l'intuition. Il y a des explications écrites destinées aux interprètes que Finnissy a choisi de ne pas reproduire dans les parties séparées. C'est le cas de certains éclaircissements concernant le fonctionnement de la notation rythmique proportionnelle dans les passages en duo (voir fig. 4), de commentaires relatifs aux indications de dynamique et de tempo (chiffre 18) ou sur le rôle des repos entre parenthèses. Comme il savait qu'il rencontrerait les interprètes, Finnissy n'a pas éprouvé le besoin d'ajouter ces détails à leurs parties respectives. Mais, sans ces informations, l'interprétation de cette pièce par un autre quatuor pourrait s'avérer problématique. Tous ces détails, et bien d'autres, sont illustrés dans un DVD qui permet au spectateur de découvrir et d'interagir avec les ressources multimédia créées lors de nos recherches sur cette pièce ${ }^{37}$.

Bénéficier d'un aperçu sur les processus de création d'interprétation et d'analyse nés de la collaboration entre compositeur et interprètes est particulièrement riche d'enseignements. Rassembler un ensemble de données à partir d'un éventail de sources aussi large que possible, puis revisiter ces données à différentes étapes, permet de retracer l'évolution d'une pièce ou d'un travail collectif. Il conviendra d'approfondir l'inspiration à la fois génétique et ethnographique de cette méthodologie afin d'en apprendre davantage sur ce que les compositeurs font et sur la manière dont ils appréhendent ce qu'ils font.

Traduit de l'anglais par Jean-Michel Magniez

37. Le site Internet <www.wlv.ac.uk/sspal/stringquartetresearch> contiendra des informations sur l'application logicielle actuellement mise au point par Bayley et Clarke. Les articles suivants décrivent l'objet et la fonction de cette application : Bayley et Clarke, "Analytical Representations of Creative Processes in Michael Finnissy's Second String Quartet », Journal of Interdisciplinary Music Studies, 3/1-2, 2009, p. 139-157, consultable à l'adresse <www.musicstudies.org/springFall2009.html> ; et « Analysing Michael Finnissy’s Second String Quartet: A Multimedia Interactive Approach », art. cit. 
AMANDA BAYLEY a soutenu à l'université de Reading une thèse sur l'interprétation des œuvres de Bartók. Elle a publié deux ouvrages collectifs chez Cambridge University Press (The Cambridge Companion to Bartók, 2001 et Recorded Music: Performance, Culture, and Technology, 2010) et des articles sur la relation entre analyse musicale et interprétation ainsi que sur le quatuor à cordes au Xxe siècle. En 2007-2009, elle a mené un projet de recherche collaboratif avec le Quatuor Kreutzer, financé par la British Academy.

Amanda Bayley, a.bayley@wlv.ac.uk

\section{Enquête sur la genèse du Deuxième quatuor à cordes de Michael Finnissy}

Cet essai porte sur le processus de composition de son Deuxième quatuor à cordes par Michael Finnissy en 2006-2007. Les sources principales de l'étude sont des entretiens semi-structurés avec le compositeur qui ont eu lieu durant une période de plus de deux ans, tant pendant qu'après la composition. L'enregistrement des interactions entre compositeur et instrumentistes a conféré une dimension supplémentaire à la saisie du processus créateur. Si le compositeur n'a pas suscité d'interventions des interprètes au cours de l'écriture, en revanche, son travail avec eux lorsqu'ils répétaient le Quatuor a produit d'importants éléments de contextualisation de ses décisions compositionnelles. Cette contextualisation est prolongée par les entretiens que nous avons menés avec Finnissy à propos de ses influences, des sources du matériau mis en œuvre, de ses méthodes de développement. Le retour avec les acteurs sur leur matériau de travail passé provoque des observations spontanées qui stimulent, chez le chercheur, de nouvelles interprétations et analyses de la musique.

This study focuses on Michel Finnissy's composition process for his Second String Quartet of 2006-2007. The main sources of this study are the semi-structured interviews with the composer that were conducted over more than two years, during as well as after the composing of the work. The recording of the interactions between the composer and the instrumentalists has given an additional dimension to the capturing of the creative process. If on the one hand the composer did not elicit any intervention from the performers during the writing process, on the other hand his work with them during rehearsals produced significant elements of contextualization of his compositional decisions. This contextualization is extended by the interviews we held with Finnissy on the subject of his influences, the sources of the material, his methods of development. Returning with the actors to their past working material provokes spontaneous observations which, for the researcher, stimulate new interpretations and analyses of music.

Diese Studie setzt sich mit dem Kompositionsprozess des Zweiten Streichquartetts von Michael Finnissy aus den Jahren 2006-2007 auseinander. Die wichtigsten Quellen für diesen Aufsatz bilden halbstrukturierte Interviews, die die Verfasserin mit dem Komponisten über einen Zeitraum von mehr als zwei Jahren sowohl während als auch nach der Komposition geführt hat. Die Aufnahme der Interaktionen zwischen dem Komponisten und den Instrumentalisten hat der Erfassung des kreativen Prozesses eine zusätzliche Dimension verliehen. Wenngleich der Komponist die Interpreten während des Schreibens zu keinerlei Mitarbeit anregte, ergaben sich aus seiner Arbeit mit ihnen während der Proben des Quartetts wichtige Kontextualisierungselemente für seine kompositorischen Entscheidungen. Diese Kontextualisierung setzt sich in den Gesprächen fort, die die Verfasserin mit Finnissy über seine Einflüsse, über die Quellen des bearbeiteten Materials und seine Entwicklungsmethoden geführt hat. Der gemeinsame Rückblick mit den Akteuren auf das bei der Arbeit verwendete Material erzeugt spontane Beobachtungen, die den Forscher zu neuen Interpretationen und Analysen der Musik anregen.
Este ensayo trata del proceso de composición, por Michael Finnissy, de su Segundo Cuarteto para cuerdas, en 2006-2007. Las fuentes principales del estudio están constituidas por entrevistas semiestructuradas con el compositor, realizadas a lo largo de un periodo de más de dos años, tanto durante como después de la composición. La grabación de las interacciones entre el compositor y los instrumentistas le otorga una dimensión suplementaria a la captación del proceso creativo. El compositor no suscitó ninguna intervención de los intérpretes durante la escritura; en cambio, su trabajo con ellos durante las repeticiones del Cuarteto produjo importantes elementos de contextualización de sus decisiones compositivas. Esta contextualización se prolonga en las entrevistas que mantuve con Finnissy acerca de sus influencias, de las fuentes del material utilizado, de sus métodos de desarrollo. La reflexión conjunta con los actores sobre los materiales de trabajo a los que han recurrido provoca observaciones espontáneas que estimulan nuevas interpretaciones y análisis de la música por parte del investigador.

Este ensaio ocupa-se do processo de composição do Segundo Quarteto de Cordas de Michael Finnissy em 2006-2007. As fontes principais do estudo são entrevistas informais com o compositor que tiveram lugar durante um período de mais de dois anos, antes e após a composição. A gravação das discussões entre compositor e instrumentistas conferiu uma dimensão suplementar à captura do processo criativo. Durante a escrita, o compositor não suscitou intervenções dos intérpretes, mas, em contrapartida, ao trabalhar com eles nos ensaios do Quarteto, produziram-se importantes elementos de contextualização das suas decisões compositivas. Esta contextualização prosseguiu com as entrevistas que efectuámos com Finnissy a propósito das suas influências, das fontes do material utilizado e dos seus métodos de desenvolvimento. $\mathrm{O}$ retorno dos actores ao material do seu trabalho passado provoca observações espontâneas que instilam no investigador novas interpretações e análises da música.

Questo saggio è dedicato al processo di composizione del secondo quartetto d'archi di Michael Finnissy nel 2006-2007. Le fonti principali dello studio sono i colloqui semi-strutturati con il compositore che hanno avuto luogo per un periodo di più di due anni sia durante sia dopo la composizione. La registrazione delle interazioni tra compositore e strumentisti ha offerto una dimensione supplementare alla comprensione del processo creativo. Il compositore non ha suscitato interventi degli interpreti nel corso della scrittura, ma il lavoro di ripetizione per l'esecuzione ha prodotto importanti elementi di contestualizzazione delle sue decisioni compositive. Questa contestualizzazione è proseguita grazie agli incontri da noi avuti con Finnissy, dove si parlato delle sue influenze, delle fonti del materiale messo in opera, dei suoi metodi di sviluppo. Ritornare con gli autori sul loro materiale di lavoro provoca osservazioni spontanee che stimolano lo studioso a nuove interpretazioni e analisi della musica 